\title{
Viewpoint
}

\section{Towards an effective and successful clinical liaison service in chemical pathology}

\author{
A O Olukoga
}

The word liaison means communication and contact between groups or units. However, implicit in this definition is the assumption that liaison between any two groups is started or maintained to achieve specific objectivesusually, for the common good. Ordinarily, in the clinical setting, liaison activities go on all the time between different units of the health care team and this should be viewed as necessary to facilitate delivery of good quality patient care. Whereas clinical liaison is inherent in the routine of all clinical disciplines, it occupies a more formal role in the business of a few, including chemical pathology (clinical biochemistry).

Traditionally, clinical liaison is considered a vital component of the duties of chemical pathology staff, both medical and scientific grade, ${ }^{12}$ and its importance has been reemphasised in the Audit Commission report. ${ }^{3}$ Liaison activities add value to laboratory service and help to ensure that the service mirrors the requirements for the clinical case mix. ${ }^{4}$ Trainees are expected to acquire experience in this aspect by a process of apprenticeship and practical application. However, despite the acknowledged importance of liaison work in chemical pathology, it has often been taken for granted and very little has been formally written about it. The ingredients of good liaison practice need to be articulated, not only for educational and training purposes but also to ensure a fairly uniform approach to the service. Personal experience and other anecdotal evidence indicate that clinical liaison services in chemical pathology vary in content, depth, style, and outcomes from one hospital to another. This brief article gives an overview of the process of clinical liaison and the tools required for an effective and rewarding practice.

Department of Clinical Biochemistry, Hope Hospital, Eccles Old Road, Salford, Manchester M6 8HD, UK
Proactive clinical liaison relates to processes initiated primarily by the laboratory and is designed to improve patient care either directly or indirectly on a continual basis. It may involve some or all of the activities listed in table 1 . These activities are facilitated by active dialogue between laboratory staff and clini- cians. Clinical problems cannot always be resolved by advice given by telephone, very often bedside consultation may be required. Abnormal results requiring follow up may be identified by different means and from various sources; these include computer generated results that are produced by delta check, reporting of tests, routine scrutiny of test results that have a high likelihood of being abnormal (for example, urgent test requests) and, less commonly, where samples have an unusual appearance (for example, lipaemia) or unusual events occur during analysis (for example, turbidity/precipitation caused by a paraprotein). Development of investigative guidelines and protocols should be undertaken jointly and mutually approved by laboratory staff and clinicians. Agreed protocols will streamline variations in clinical practice and facilitate a cost effective service. A newsletter, preferably across all pathology disciplines, may be published at regular intervals and directed mainly at general practitioners. It should serve as a medium for continuing education in laboratory medicine and disseminating information on on-going initiatives to improve quality of laboratory services.

\section{REACTIVE}

Reactive clinical liaison concerns activities undertaken by the laboratory in response to extra-laboratory queries and informal clinical questions, raised usually by clinicians. Action taken will depend on the nature of the query and may involve any of the items listed under proactive liaison.

\section{Staff involved with clinical liaison}

Clinical liaison is accomplished chiefly by chemical pathologists and clinical scientists. The chemical pathologist, a medical graduate, with a broad clinical background combined with specialist training in the clinical and laboratory aspects of a wide range of medical conditions, is ideally suited to undertake bedside consultation, which is often necessary to resolve clinical problems and, sometimes, to advise on treatment. These activities are complemented by those of the clinical scientist who, while of a science background, develops clinical awareness often in chosen areas of spe- 
Table 1 Activities undertaken in the proactive mode of clinical liaison

Telephoning to discuss results and patients, and advising on further tests

Development of test repertoire

Visits to wards and other clinical units on an ad hoc or regular basis

Attending ward rounds/clinical team meetings

Appending interpretative comments to reports

Development of investigative protocols

Clinical audit (multidisciplinary)

Regular joint educational meetings with hospital clinicians and general practitioners

Newsletter

Table 2 Benefits of a successful clinical liaison service

Improved patient care

Opportunity to identify and document instructive clinical cases

Potential source of research questions

Enhancement of the profile of individual laboratory staff and that of chemical pathology as a clinical discipline

Personal education for both the laboratory staff and clinicians

cialist interest. Considering that the professional inclinations of staff vary, it is to be expected that their ability to tackle specialist, complex clinical issues will also vary. It is, therefore, vitally important that individual limitations are recognised and appropriate assistance from other colleagues sought when necessary.

\section{Attributes and skills required for clinical liaison}

Clinical liaison is an art and good practice demands the right approach and appropriate skills. The following are some of the qualities necessary to ensure a successful liaison service:

- Knowledge-in depth understanding of the various aspects of chemical pathology and wide appreciation of clinical medicine

- Organisational insight-into the home department, hospital and regional as well as national laboratory networks

- Effective communication skills

- Appropriate temperament-confidence, patience, and tact

- Awareness of professional concerns-be familiar with the local hospital professional interrelationships and appropriate personality issues

- Maintaining good rapport-with clinical colleagues and other professionals in the health care team

- Commitment to continuing personal education

- Participation in educational activities of the local hospital.

\section{Hindrance to effective clinical liaison}

Potential impediments to an effective clinical liaison process may arise as a result of organisational or staffing difficulties in the following broad categories:

- Laboratory

- Clinical units

- Infrastructure

- Structural layout of the hospital.

Laboratory staff

Lack or deficiency of personnel with appropriate skill levels.

Clinicians

Poor interprofessional links resulting from lack of understanding of the laboratory role, lack of personal level contact or delineation and truncation of professional roles.

\section{Infrastructure}

A functional organisational set up complete with a good information system, computerisation, cross discipline educational programmes, and resource units enhance clinical liaison; where these are deficient or unavailable the process may be hindered.

\section{Structural layout of the hospital}

The layout of the hospital may be such that the laboratory is physically isolated and distant from the clinical areas. This arrangement does not facilitate easy movement or interaction.

\section{Mechanisms for minimising obstacles to clinical liaison}

The following are some of the means of ensuring a smooth and rewarding clinical liaison practice:

- Acquisition of the necessary attributes and skills

- Securing the respect and confidence of clinician colleagues by demonstrating in depth knowledge; providing timely, constructive and helpful advice; and approaching issues as a team member

- Liaise initially with the junior staff whenever appropriate

- Being sensitive to the circumstances at the point of liaison; choose the right moment and place to discuss the clinical matters

- Accepting criticisms in good faith and offering constructive response to them.

\section{Gains of effective and successful clinical liaison}

Some of the potential benefits of clinical liaison are highlighted in table 2.As far as health care delivery is concerned the ultimate outcome of any clinical process should be improvement in patient care. Unfortunately, there is a paucity of objective evidence showing the impact on the quality of patient care of clinical liaison work in chemical pathology. However, an audit conducted recently in our hospital showed a threefold improvement in the appropriate investigation of patients with moderate and severe hyponatraemia as a direct result of chemical pathology liaison practice. ${ }^{5}$ In addition chemical pathology medical staff advised on the treatment of some of these patients. Another audit from our group showed clearly that proactive liaison activity by laboratory staff was instrumental in ensuring that patients who presented to the accident and emergency department with suspected myocardial infarction and previously unrecognised dyslipidaemia were identified promptly and followed up for secondary prevention.

Regarding primary care, there is some evidence that general practitioners demand, use, and are generally pleased to have access to laboratory staff with the appropriate skill level who can advise on biochemical investigations and interpret the results. ${ }^{7}$ This may also involve advice on management of metabolic problems including lipid disorders. This partnership, 
therefore, has a positive influence on the quality of care that general practitioners provide their patients. Conceivably, the requirement for laboratory staff to be available to provide advice on clinical and other laboratory matters to clinicians, both during normal working period and out of hours, will increasingly become a formal component of service level agreements for laboratory work.

The medical literature is replete with a large number of instructive clinical case reports that are products of collaboration between laboratory staff and clinicians. This is another instance of the benefit of clinical liaison. The joint input not only ensures that such cases have been adequately and appropriately worked up clinically and scientifically but also enables a balanced presentation. Suffice it to say that case reports published by laboratory staff represent only a small proportion of the clinical material that passes through the department. It has been suggested that chemical pathologists who follow up all relevant material coming through their department will gain vast experience in metabolic disorders, perhaps more in some areas than any one clinician. $^{8}$

Clinical liaison provides opportunities for mutual education of laboratory staff and clinicians. Given time clinicians learn to make the most appropriate use of the laboratory ${ }^{9}{ }^{10}$ while laboratory staff adapt delivery of laboratory services to meet the changing needs of the clinical service. In this way an efficient and cost effective use of resources is ensured. Furthermore, in hospital practice, during the course of clinical liaison, regular interaction of chemical pathologists with house staff and other junior clinical staff in general professional training should provide such trainees with continuing education in applied clinical biochemistry. Hopefully, this should also stimulate their interest in the subject enough to consider it as a career choice.

\section{Conclusion}

Clinical liaison is an important aspect of chemical pathology services that enhances the quality of patient care and facilitates mutual education of laboratory staff and clinicians. Like any clinical process, successful clinical liaison requires the right skills and attributes. To be of any benefit these skills and attributes need to be articulated by good communication. Furthermore, additional objective evidence of the benefits of clinical liaison is required to consolidate its importance.

I am grateful to my colleagues, Dr V E F Crowley, Dr G Horsman, Dr M F Stewart and Prof P Vadgama for their comments and helpful discussions.

1 Department of Health and Social Security. Hospital laboratory services. London: HMSO, 1970. (HM (70) 50).

2 Baron DN. Training and organisation for pathology in Britain: the role of chemical pathologists. Am $\mathcal{F}$ Clin Pathol 1975;63:269-75.

3 Audit Commission. Critical Path: an analysis of the pathology services. London: HMSO, 1993.

4 Barth J. The chemical pathologist as clinician. Bulletin of the Royal College of Pathologists 1995;89:19-20.

5 Crowley VEF, Olukoga AO, Fielding G, McMurray JR Stewart MF. Audit of laboratory-based investigation of moderate and severe hyponatraemia $(\mathrm{Na}<125 \mathrm{mmol} / \mathrm{L})$ in Hope Hospital [abstract]. Proceedings of the XVI International Congress of Clinical Chemistry. London, 8-12 July 1996;A510:174.

6 Olukoga AO, Crowley VEF, Driscoll P, Barber D, Weinkove C, McMurray JR, et al. The role of the laboratory in initiating cholesterol measurement in patients with myocardial ing cholesterol measurement in patients with myocardial infarction [abstract]. Proceedings of the XVI International Congress of

7 Allen KR, Harris CM. Measure of satisfaction of general practitioners with the chemical pathology services in Leed Western Health District. Ann Clin Biochem 1992;29:331-6.

8 Zilva JF. What are clinical chemists for? Bulletin of the Royal College of Pathologists 1996;96:18-19.

9 Gama R, Nightingale PG, Broughton PMG, Peters M, Ratcliffe JG, Bradby GVH, et al. Modifying the request behaviour of clinicians. $\mathcal{F}$ Clin Pathol 1992;45:248-9.

10 Sandle LN. How do we stop doing clinically irrelevant tests [abstract]? Proceedings of the XVI International Congress of Clinical Chemistry. London, 8-12 July 1996;A521:177. 